\title{
CONDITIONAL EXPECTATIONS OF RANDOM LINEAR OPERATORS IN BANACH SPACES
}

\author{
PATrycja Jędrzejewska, Katarzyna Majcherek And August ZapaŁa
}

Abstract. The paper generalizes in various ways factorization property, known till now only for real-valued random variables, to conditional expectations of random linear continuous transformations in Banach spaces measurable with respect to sub- $\sigma$-fields. We investigate compositions of random linear operations formed by means of outer and inner maps measurable with respect to sub- $\sigma$-fields and prove that under suitable assumptions such operations can be pulled out of the conditional expectation operator.

Mathematics subject classification (2010): 60H25, 60B11, 47B80, 46B09, 47A56.

Keywords and phrases: conditional expectation in a Banach space, essentially separably valued random element, random linear operator.

\section{REFERENCES}

[1] G. Androulakis, K. Beanland, S. J. Dilworth And F. SAnacory, Embedding $\ell_{\infty}$ in the space of bounded operators on certain Banach spaces, Bull. London Math. Soc., 38 (2006), 979-990.

[2] J. B. Conway, A Course in Functional Analysis, Springer, 2nd ed., New York, (1997).

[3] J. Diestel and J. J. Uhl, Vector Measures, Math. Surveys No 15, Amer. Math. Soc. Providence, Rhode Island (1977).

[4] N. N. Vakhania, V. I. Tarieladze and S. A. Chobanyan, Probability Distributions on Banach Spaces, D. Reidel, Dordrecht, Boston, Lancaster, Tokyo, (1987).

[5] A. M. ZAPAŁA, Jensen's inequality for conditional expectations in Banach spaces, Real Analysis Exchange, 26 (2) (2000/2001), 1-12. 